\title{
Abordagem Baseada em Aprendizado de Máquina para Detecção de Alarmes Industriais
}

\author{
Emerson Vilar de Oliveira* Yuri Thomas Pinheiros Nunes* \\ Luiz Affonso Guedes* \\ * Programa de Pós Graduação em Engenharia Elétrica e de \\ Computação, Universidade Federal do Rio Grande do Norte, RN, \\ (e-mail: emerson_vilar@hotmail.com).
}

\begin{abstract}
Alarm systems are a great ally in monitoring industrial processes. They serve to alert operators about deviations from the specifications. The techniques most used in the tuning of alarms make use of several parameters and specific knowledge about the process to detect such failures. A new approach making use of machine learning methods to detect patterns in the process that should be pointed as deviation is described in this paper. For this, a model is trained to determine the state of the system by making use of process data and state feedback. The proposed approach is applied to a case study of monitoring a simulated industrial process, the Tennessee Eastman Process.

Resumo: Os sistemas de alarmes são um grande aliado no monitoramento de processos industriais. Eles servem para alertar aos operadores sobre desvios nas especificações. As técnicas mais usadas na sintonia de alarmes fazem uso de diversos parâmetros e de conhecimentos específicos sobre o processo para detectar tais falhas. Uma nova abordagem fazendo uso de métodos de aprendizado de máquina para detectar padrões no processo que devem ser apontados como desvio é descrita neste trabalho. Para isso um modelo é treinado para determinar o estado de sistema fazendo uso dos dados do processo e realimentação dos estados. A abordagem proposta é aplicada para em um estudo de caso de monitoramento de um processo industrial simulado, o Tennessee Eastman Process.
\end{abstract}

\section{Keywords: Industrial Alarms; Applications of Intelligent Systems in Automation; Machine Learning. \\ Palavras-chaves: Alarmes Industriais; Aplicações de Sistemas Inteligentes em Automação; Aprendizagem de Máquinas.}

\section{INTRODUÇÃO}

O sistema de alarme é parte integrada dos sistemas modernos de monitoramento computadorizado, como os sistemas de controle distribuído e controle, supervisão e aquisição de dados (Wang et al., 2016). Um sistema de alarmes é definido como "a coleção de hardware e software que detecta um estado de alarme, comunica a indicação desse estado aos operadores e registra mudanças no estado de alarme" (ANSI/ISA, 2009a). Um alarme é uma sinalização (visual e ou audível) que indica à operação do processo que alguma ação operacional deve ser tomada (ANSI/ISA, 2009a).

Devido à complexidade da operação de plantas industriais modernas, sistemas de alarmes tornaram-se fundamentais para sua segurança operacional. Sendo que o mau funcionamento de sistemas de alarmes podem causar acidentes com perdas de vidas, danos ao meio ambiente e prejuízos econômicos. Os sistemas de alarmes são a ferramenta

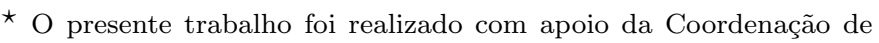
Aperfeiçoamento de Pessoal de Nível Superior - Brasil (CAPES) Código de Financiamento 001
}

mais importante para os operados na hora de monitorar a segurança da planta. Se alguma falha que comprometa o funcionamento seguro da fábrica ocorre, um ou mais alarmes são acionados e cabe ao operador tomar alguma medida corretiva para tornar o processo a sua condição normal (Bransby and Jenkinson, 1998).

Alguns acidentes ratificam essa realidade, como o caso de explosão de uma planta de refino de petróleo da British Petroleum Refinery na Cidade do Texas (USA) em 2005, que fez além de vítimas fatais, muitos feridos e um dano econômico de aproximadamente 1,5 bilhão de dólares em um ano (Manca and Brambilla, 2012). Também em 2005, podemos citar a explosão de uma usina petroquímica na província de Jilin (China), que além dos danos causados pela explosão, despejou cerca de 100 toneladas de substâncias tóxicas no Rio Songhua (Zhang et al., 2010).

A ocorrência de desastres de tais magnitudes mostraram a importância de um método formal para configuração dos alarmes. Isso ganhou força na comunidade científica e industrial, gerando a criação de algumas normas e guias 
de boas práticas no uso de sistemas de alarmes, como a EEMUA 191 e ANSI/ISA 18.2.

As abordagens mais simples para detecção de alarmes se baseiam no uso de thresholds para determinar quando um sinal desvia de sua operação normal. O uso do threshold possibilita repetitivas transições no sinal de alarme em um curto intervalo de tempo caracterizando chattering alarms (Adnan et al., 2011). Existem ainda casos de fleeting alarms onde o sinal de processo oscila bruscamente e rapidamente volta ao estado anterior (ANSI/ISA, 2009b). Para lidar com estes problemas fazem-se uso de deadbands e delay timers. O uso de deadbands é indicado para diminuir a incidência de chattering alarms, principalmente relacionados a ruído. Para remoção de fleeting alarms o uso de delay timers é mais indicado por necessitar que o sinal apresente o indicativo de alarme de maneira contínua.

Métodos como detecção de outliers $(3 \sigma)$ e otimização de desempenho (Xu et al., 2012) são técnicas para determinar thresholds de alarmes. Similarmente existem técnicas para determinar o deabdand como análise de séries temporais (Hugo, 2009) e uma extensão de otimização de desempenho (Naghoosi et al., 2011). Outras técnicas usam as durações e intervalos entre alarmes para remoção dos que se repetem, fazendo uso de delay timers (Wang and Chen, 2014). A correta configuração dos parâmetros de alarme é uma das deficiências dos sistemas de alarme (Wang et al., 2016). Além de definir corretamente os valores dos parâmetros é importante saber quando usar os recursos de deadband e delay timer.

É proposto no presente trabalho, uma nova abordagem para detecção de alarmes fazendo uso de um sistema realimentado e de técnicas de aprendizado de máquina. A saída do sistema é o próprio sinal de alarme. Além da realimentação do sinal de alarme o sistema também conta com dados da variável de processo como entrada. O objetivo é diminuir o número de parâmetros usados e otimizar as métricas de desempenho. A abordagem foi testada experimentalmente utilizando o simulador de processos Tennessee Eastman Process.

Inicialmente, a Seção 2 introduz conceitos básicos necessários para situar o problema. Em seguida, na Seção 3 é mostrada a proposta do sistema de alarmes, como também, quais estruturas e métodos são utilizados no seu funcionamento. A Seção 4 traz a aplicação do sistema em testes feitos utilizando o Tennessee Eastman Process para a simulação de um processo industrial. Por fim, a Seção 6 apresenta as conclusões obtidas e objetivos para trabalhos futuros.

\section{ALARMES INDUSTRIAIS}

Nesta seção serão apresentados os conceitos sobre alarmes industriais, seus parâmetros de sintonia típicos, problemas e métricas de desempenho, como FAR, $M A R, A A D$ e chattering index.

\subsection{Alarmes: Definições e Problemas Típicos}

Um alarme é modelado matematicamente como um sinal binário. Normalmente o valor 0 é associado ao alarme inativo enquanto o valor 1 ao alarme ativo. A definição entre ativo e inativo se faz utilizando uma função de decisão $f(\cdot)$. A equação (1) descreve um alarme $x_{a}$ em função de $t$, definido para a variável de processo $x(t)$ utilizando uma função de decisão $f_{x}(\cdot)$.

$$
x_{a}(t)=f_{x}(x(t))
$$

A função de decisão mais simples define um limite que deve ser respeitado por uma variável de processo $x$. Esse limite é conhecido como threshold. A equação (2) dá um exemplo dessa estratégia.

$$
f(x(t))=x(t)>x_{t p}
$$

onde $x(t)$ é referente a variável de processo no instante $t \mathrm{e}$ $x_{t p}$ ao threshold do alarme.

Existem dois tipos básicos de alarmes derivados do uso de thresholds: os alarmes do tipo high (alto) e low (baixo). As equações (3) e (4) definem suas funções de decisão.

$$
\begin{aligned}
& x_{H}(t)=x(t)>x_{t p} \\
& x_{L}(t)=x(t)<x_{t p}
\end{aligned}
$$

onde, $x_{H}$ e $x_{L}$ são alarme high e alarme low respectivamente. O threshold para ativação dos alarmes é $x_{t p}$.

Problemas comuns associados ao uso de thresholds na detecção de alarmes são chattering alarms e fleeting alarms. Os chatterings alarms ocorrem quando a variável de processo atua próximo do limiar de ativação. Esse comportamento pode causar diversas ativações e desativações consecutivas do sinal de alarme em um curto intervalo de tempo. Entretanto, quando a variável de processo está suficientemente distante do threshold, alarmes podem ocorrer por presença de ruídos ou erros de leitura. Nesses casos os alarmes têm curta duração e não há repetição, caracterizando fleeting alarms.

Para contornar estes problemas, faz-se uso de estratégias como delay timers ou deadbands em conjunto com o threshold. Essas estratégias fazem uso de funções de decisão mais elaboradas para garantir a qualidade do sinal de alarme. O uso de delay timers é indicado para remover alarmes incômodos de curta duração, como os fleeting alarms. Para tratar os chattering alarms é mais indicado o uso de deadbands, por gerar uma barreira para desativação próxima ao threshold.

O delay timer impõe que a variável de processo apresente um número mínimo de amostras que desviem do threshold consecutivamente. O sinal de alarme mantém seu valor anterior caso a variável de processo não apresente tal comportamento. A equação (5) descreve a estratégia de delay timer.

$$
f\left(x_{a}\left(t^{-}\right), x(t), \cdots, x(t-n)=\left\{\begin{array}{c}
1, \prod_{i=0}^{n} x(t-i)>x_{t p} \\
0, \prod_{i=0}^{n} x(t-i)<x_{t p} \\
x_{a}\left(t^{-}\right), \text {c.c. }
\end{array}\right.\right.
$$

onde, $x_{a}\left(t^{-}\right)$represente o valor passado do sinal de alarme, o produtório em $n$ representa os $n$ instantes em que variável de processo apresenta o mesmo comportamento em relação ao threshold, $x_{t p}$.

$\mathrm{O}$ uso de deadbands consiste em adicionar um limiar para dificultar a transição do sinal alarme. Esse novo limiar, $x_{d b}$, é utilizado para desativar o alarme. No caso de um 
alarme $h i g h, x_{d b}$ é inferior ao $x_{t p}$, criando uma zona onde o valor do sinal de alarme depende do seu estado passado. A equação (6) demonstra tal estratégia.

$$
f\left(x(t), x_{a}\left(t^{-}\right)\right)=\left\{\begin{array}{c}
1, x(t)>x_{t p} \\
0, x(t)<x_{d b} \\
x_{a}\left(t^{-}\right), c . c .
\end{array}\right.
$$

Dessa maneira podemos considerar que um alarme tipico possui até três parâmetros: o threshold, o tamanho do delay timers ( $n$ amostras) e o limiar de desativação $\left(x_{d b}\right)$. Entretanto, é incomum o uso dos três parâmetros simultaneamente. Apesar das normas e guias possuírem valores iniciais recomendados para estes parâmetros, ainda é necessário um ajuste fino que demanda uma análise específica para cada parâmetro.

\subsection{Desempenho de Sistemas de Alarmes}

Nesta seção serão apresentadas as métricas de desemprenho para um sistema de alarmes. O objetivo de tais métricas, é medir a qualidade do sistema de alarmes.

Considerando uma variável de processo em tempo discreto $x(t)$ e um threshold $x_{t p}$ associado a ela, dois tipos indesejados de alarmes podem aparecer. Falso alarme é aquele que é gerado quando a variável de processo está em sua região normal. Alarme perdido é quando a variável de processo está se comportando anormalmente e nenhum alarme é gerado (Xu et al., 2012). Supondo que as funções de densidade de probabilidade das regiões normal e anormal da variável $x$ são conhecidas, a região normal foi definida como $q(x)$ e a anormal como $p(x)$. A Figura 1 expressa todos esses elementos graficamente.

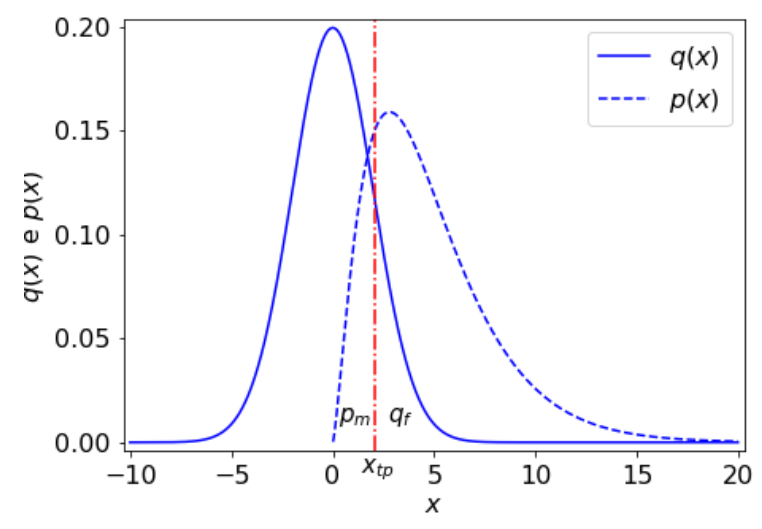

Figura 1. As FDPs das regiões normal e anormal de $x$.

O FAR (False Alarm Rate), é definido como a probabilidade de falsos alarmes ocorrerem na área sob a curva da distribuição $q(x)$, para valores de $x$ maiores que $x_{t p}(\mathrm{Xu}$ et al., 2012). FAR está indicado na Figura 1 como $q_{f}$.

$$
F A R=\int_{x_{t p}}^{+\infty} q(x) d x
$$

Essa métrica expressa o quanto o sistema indicou que havia alarmes, sem de fato existir uma anormalidade presente.

O MAR (Missed Alarm Rate) é definido como a probabilidade de alarmes perdidos ocorrerem na área sob a curva da distribuição $p(x)$, para valores de $x$ menores que $x_{t p}$ (Xu et al., 2012). MAR está indicado na Figura 1 como $p_{m}$

$$
M A R=\int_{-\infty}^{x_{t p}} p(x) d x
$$

Essa métrica expressa o quanto o sistema falhou em indicar um alarme, quando de fato se deveria acionar.

O $A A D$ (Averaged Alarm Delay) é definido como o valor esperado de $T d$, onde $T d$ é a diferença de tempo entre o instante $t_{0}$, em que a variável de processo sai da sua região normal e o instante de tempo $t_{a}$, em que de fato o alarme é acionado (Xu et al., 2012).

$$
\begin{gathered}
T d=t_{a}-t_{0} \\
A A D=E(T d)
\end{gathered}
$$

O $A A D$ pode ser interpretado como um atraso de ativação, ou seja, uma medida de quanto o sistema demora a indicar um alarme, quando de fato há uma ocorrência.

Chattering alarms se referem aos alarmes que fazem repetidas transições entre alarme e não alarme em um curto intervalo de tempo, sem uma devida resposta dos operadores (Wang and Chen, 2014). Índices de chattering (CI) para alarmes foram definidos ao longo dos anos. Alguns são baseados em run e run length, que no contexto de alarmes são estruturas que medem as amostras (ou tempo) entre dois alarmes consecutivos. Neste artigo será usado o índice definido em Wang and Chen (2013), que pode ser expresso por:

$$
\eta=\frac{2 \sum_{r} A C_{r} / r}{N}
$$

Onde $r$ é o run length, $A C_{r}$ é quantidade total de vezes que aquele run length em específico ocorreu, $N$ é o número de amostras totais do sinal de alarme e o inteiro 2 aparece como um fator de escala para a variação do índice (Wang and Chen, 2013).

\section{METODOLOGIA}

Nesta seção será apresentada a metodologia da abordagem proposta. O fluxo de informação e de quais estruturas ele faz uso para inferir o sinal de alarme.

Como discutido na Seção 2, o uso de delay timers e deadbands apresenta obstaculo para o sinal de alarme mudar de estado. Este obstaculo pode depender apenas do estado atual do processo e do alarme, no caso do deadband. $\mathrm{O}$ uso de delay timers apresenta a necessidade de monitorar $n$ amostras passadas do processo e o estado atual do sinal de alarme. Os sistemas de alarmes atuais realizam a inferência do sinal de alarmes baseadas em lógicas simples e claras.

A abordagem proposta tem o intuito de integrar essas dependências do estado do alarme e processo. Na Figura 2 podemos apontar os três componentes principais dos sistemas de alarmes atuais: os dados de processo, os dados de alarme e uma máquina de inferência. Nesse modelo, os dados de alarme são realimentados e usados conjuntamente com os dados de processo na inferência do sinal alarme. 


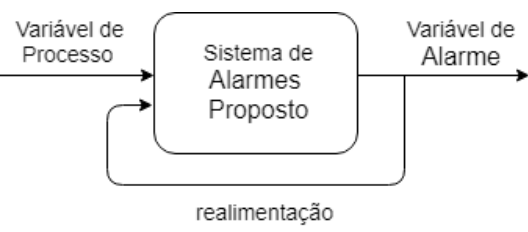

Figura 2. Sistema de Alarmes proposto.

Os dados de processo podem ser modelados como um vetor das últimas $n$ amostras, incluindo o valor atual do processo. A equação (12) define a entrada dos dados de processo, $w_{v a r}$, de uma dada variável de processo, $x_{p}(t)$.

$$
w_{v a r}=\left[x_{p}(t-n+1), x_{p}(t-n+2), \cdots, x_{p}(t)\right]
$$

Os dados de alarme podem ser modelados de maneira análoga aos dados de processo. A realimentação de dados de alarme, $w_{a l r}$, é composta pelas $m$ amostras mais recentes do sinal de alarme, exceto o valor atual que será inferido. Ela não possui o valor atual da variável de alarme, já que o mesmo, é o objetivo a ser alcançado pelo sistema. A equação (13) define $w_{\text {alr }}$ em função de um sinal de alarme, $x_{a}(t)$.

$$
w_{a l r}=\left[x_{a}(t-m), x_{a}(t-m+1), \cdots, x_{a}(t-1)\right]
$$

A máquina de inferência proposta possui uma entrada, $w_{\text {ent }}$, que é composta pelas demais entradas, $w_{v a r}$ e $w_{a l r}$, descrita na equação (14).

$$
w_{\text {ent }}=\left[w_{\text {var }}, w_{\text {alr }}\right]
$$

onde $w_{\text {ent }}$ tem o tamanho de $(n+m)$ amostras.

O objetivo de um sistema de alarmes é detectar se houve alguma mudança no comportamento padrão da variável de processo. Para isso, é necessário algum método que observe esses padrões e os reconheça. O aprendizado de máquina é um subcampo da ciência da computação que evoluiu do conceito de reconhecimento de padrões. Essa habilidade que os algoritmos de aprendizado de máquina tem, de aprender sem serem explicitamente programados para determinado objetivo, é um dos fatores que os levam a serem escolhidos como modelo. Eles operam construindo o tal modelo, de modo a fazer previsões ou decisões, guiados pelos dados e não simplesmente seguindo instruções inflexíveis predefinidas.

Então o algoritmo de aprendizado de máquina escolhido será treinado. Ao receber um novo ponto, ele tem que ser capaz de decidir a qual grupo aquele novo ponto pertence, para que em outro momento seja feita as predições para o valor de alarme. Cada janela de entrada é interpretada como um dos novos pontos a serem inseridos na máquina. $\mathrm{Na}$ fase de treinamento, o algorítimo é responsável por agrupar e rotular todos esses pontos, de modo a separá-los em dois grupos, onde um é referente a região normal da variável de processo e o outro a região anormal. $\mathrm{Na}$ fase de predição, ele rotula o novo ponto, indicando a qual grupo ou classe o respectivo ponto pertence.

Algumas alterações na construção das estruturas são feitas para a fase de predição do alarme. Nessa etapa, se tem uma janela $w_{v a r}$ da variável de processo referente aquele instante de tempo, com o objetivo de inferir o valor de alarme a partir do que foi aprendido na fase de treino. Inicialmente não se tem nenhuma informação ou valor de alarme para se inserir na primeira janela de alarme $w_{\text {alr }}$. Assumindo que o valor do alarme inativo é 0 e a variável de processo inicia sem anormalidades, a janela $w_{\text {alr }}$ será populada com um vetor de $m$ zeros. A $w_{v a r}$ e a $w_{\text {alr }}$ compõe assim a primeira entrada $w_{\text {ent }}$ do sistema. Os valores dos alarmes serão realimentados para a realização de novas predições.

Como previamente comentado, o valor previsto pela máquina, é a qual aglomerado aquele ponto de entrada pertence. Expresso em notação binária, o 0 (grupo 0) é interpretado como o caso em que a entrada esteja em região normal, enquanto o 1 (grupo 1) é o caso em que a entrada se encontra em região anormal.

Por fim, os valores previstos pela maquina em cada instante de tempo, vão formar a variável de alarme $x_{a}(t)$.

Os tamanhos das janelas $n$ e $m$, podem ser definidos empiricamente de acordo com a qualidade de treinamento do modelo escolhido. Como dito anteriormente, aqui, os valores obtidos nas métricas de avaliação servirão como critério para se definir os valores mínimos de $n$ e $m$, tornando a comprimento das janelas, os únicos parâmetros passíveis de ajustes para uma otimização do sistema.

\section{EXPERIMENTOS}

Nesta seção está descrita a elaboração dos experimentos. Inicialmente será discutido o simulador Tennessee Eastman Process usado para gerar os dados e construir os brancos de treinamento e teste. Aqui também serão mostrados os critérios usados para geração de alarmes que servirão para o treinamento e para as métricas de avaliação. A Seção é finalizada discutindo o modelo escolhido para os experimentos, o K-means.

\subsection{Tennessee Eastman Process}

O Tennessee Eastman Process (TEP) é um simulador de um processo industrial baseado em um processo real (Downs and Vogel, 1993). Composto por quatro reagentes, um componente inerte, um subproduto e dois produtos. Esse processo é controlado por 12 variáveis manipuladas, contendo um total de 41 variáveis medidas, destas, 22 são continuas e 19 amostradas. Para os experimentos, apenas variáveis continuas serão utilizadas. O TEP possui 20 distúrbios, dos quais 3, serão utilizados juntos no banco de treinamento e posteriormente testados separadamente utilizando o banco de testes.

\subsection{Geração de Dados}

Para o banco de treino, foram simuladas aproximadamente 920 horas de operação do processo, com período de amostragem de 36 segundos, totalizando 92024 amostras das variáveis de processo. A simulação também contou com ocorrências aleatórias dos distúrbios 1, 2 e 6, onde apenas um distúrbio ocorria por vez, não havendo sobreposição dos mesmos. A Tabela 1 expressa em amostras a sequência que foi gerada.

As colunas da Tabela 1 representam respectivamente: o identificador do distúrbio, o índice da amostra em que ele iniciou, o índice da amostra no qual ele terminou e a quantidade de amostras em que o distúrbio permanece 
ativo. O sinal inicia e finaliza sem nenhum distúrbio ativo. Entre o fim e o começo de cada distúrbio existe uma região sem a presença de nenhuma anormalidade. As regiões sem anormalidade tem duração aleatória entre 5000 e 7000 amostras uniformemente distribuídas.

A Figura 3 ilustra o comportamento da variável de processo de nome XMEAS10, gerada no TEP, para a sequência de distúrbios apresentada na Tabela 1.

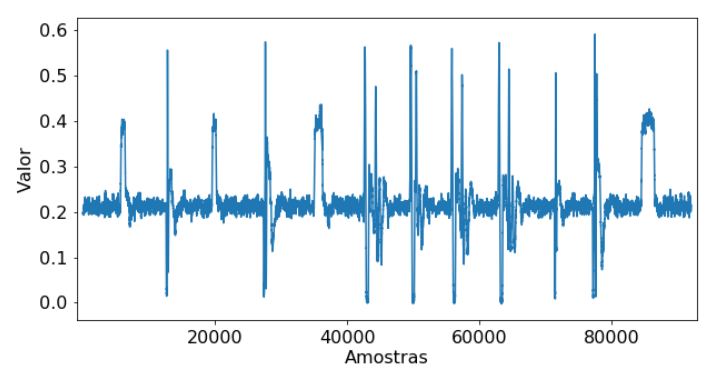

Figura 3. Variável XMEAS10 usada no treinamento.

\subsection{Banco de Testes}

Para validar a abordagem é necessário testar o sistema treinado em outro conjunto de dados. Diferentemente do treinamento, os testes são feitos para os distúrbios separadamente, ou seja, existe um banco de testes com ocorrências do Distúrbio 1, outro para o Distúrbio 2 e outro para o Distúrbio 6. Os distúrbios iniciam e terminam em amostras aleatórias e só ocorrem um por vez.

Nos bancos de testes foram inseridos comportamentos que se assemelham a causas de fleeting alarms. Esses pontos consistem de 5 amostras consecutivas em regiões sem e com alarmes esperados. O objetivo é avaliar a robustez da abordagem a um comportamento indesejado não presente no conjunto de treinamento.

\subsection{Critérios de Determinação de Alarmes}

O treinamento dos modelos de aprendizado de máquina necessita que os dados representem os cenários reais de aplicação. Assim, os dados de processo foram analisados para determinar as regiões que devem produzir alarmes relevantes para os operadores. Os sinais de alarme definidos consideram que um alarme deve ser ativo tão logo a variável de processo inicie um desvio perceptível. Analogamente, as desativações de alarme devem considerar o retorno do processo à normalidade.

Tabela 1. Ocorrência dos Distúrbios.

\begin{tabular}{cccc} 
Distúrbio & Início & Fim & Duração \\
\hline 2 & 5637 & 6297 & 660 \\
1 & 12562 & 12731 & 169 \\
2 & 19479 & 20070 & 590 \\
6 & 27297 & 27552 & 254 \\
2 & 34950 & 36147 & 1197 \\
1 & 42515 & 44123 & 1607 \\
1 & 49469 & 50311 & 841 \\
1 & 55684 & 57172 & 1487 \\
1 & 62816 & 64264 & 1448 \\
6 & 71365 & 71494 & 128 \\
6 & 77130 & 77442 & 312 \\
2 & 84478 & 86414 & 1935 \\
\hline
\end{tabular}

A Figura 4 ilustra o comportamento da variável XMEAS10 ao Distúrbio 2. São expressas a variável de processo, a região em que o distúrbio está ativo e a região de alarme associada. Percebe-se que o alarme é definido como ativo depois do começo da anormalidade e desativo após o fim da região de anormalidade. Isso se dá devido aos atrasos inerentes do processo e ao que se define como alarme.

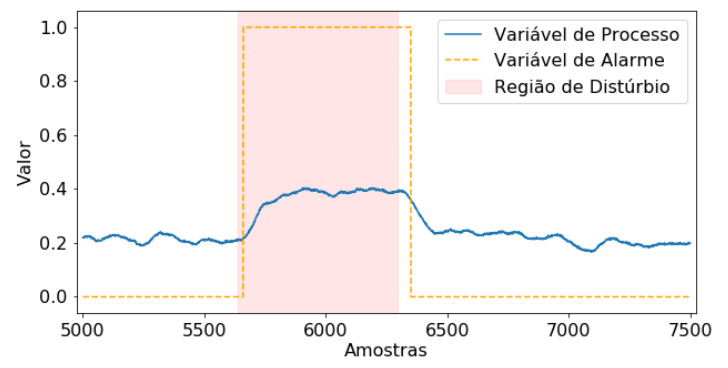

Figura 4. Região de alarme da variável XMEAS10 para Distúrbio 2.

Em alguns casos, não é possível inferir nenhuma região de alarme para aquela ocorrência de distúrbio. A Figura 5 ilustra a variável de processo XMEAS10 com a ocorrência do Distúrbio 1. Para este caso, a variável sendo vista isoladamente por um intervalo de tempo maior, demonstra um comportamento de estabilização durante a ocorrência do distúrbio, o que causaria uma ativação e desativação de um alarme, sem necessidade de um ação do operador.

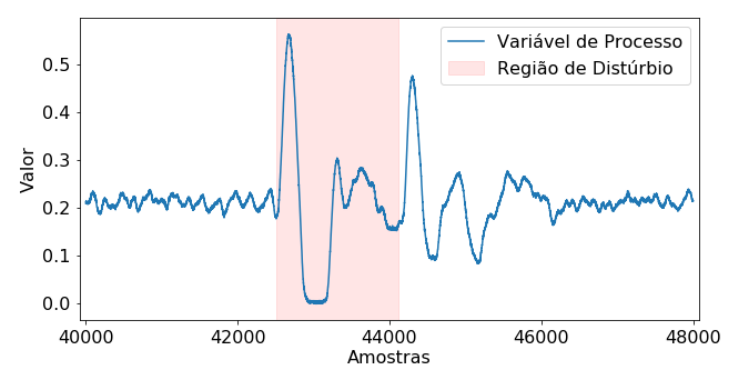

Figura 5. Comportamento da Variável XMEAS10 para Distúrbio 1.

Os sinais de alarmes do banco de treino foram gerados atendendo a esses critérios. A Figura 6 mostra a Variável de Processo XMEAS10 e sua região de alarme associada.

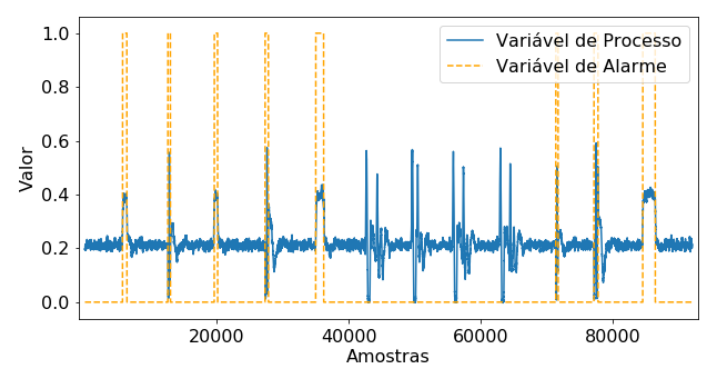

Figura 6. Variável XMEAS10 e sua respectiva região de alarme. 


\subsection{Modelo de treinamento: K-means}

O método de aprendizado escolhido é um clássico algoritmo para clusterização, o K-means. Algoritmos de clusterização procuram aprender, usando propriedades dos dados usados no treinamento, uma separação ótima ou uma rotulação dos grupos de pontos (VanderPlas, 2016). O Kmeans, procura por um predeterminado número de clusters dentro do conjunto de dados, usando uma concepção de como é o clustering ideal.

- O "centro de cluster" é a média aritmética de todos os pontos pertencentes ao cluster.

- Cada ponto está mais próximo do centro do seu próprio cluster do que do centro de outro cluster.

Essas duas suposições são a base do modelo K-means (VanderPlas, 2016).]

Devido a características do modelo escolhido é necessário balancear e normalizar os dados. O balanceamento consiste em igualar o número de amostras em região de alarme e não alarme para evitar enviesamento do treinamento. Caso existam mais pontos em alguma das regiões, é possível que essa diferença altere o centro de cluster para algum outro ponto não fidedigno ao comportamento da variável. Também é necessário normalizar os dados da variável de processo devido ao $K$-means ser sensível às magnitudes dos dados. É importante que ambos os passos sejam feitos com escolha de pontos aleatórias para ambas as regiões, mas obedecendo à distribuição original da variável. Após a aplicação desses passos, na ordem em que foram apresentados, as variáveis de processo e alarme estão devidamente ajustadas para passarem pelo treinamento.

A Figura 7 mostra a Variável XMEAS10, ajustada para a fase de treinamento.

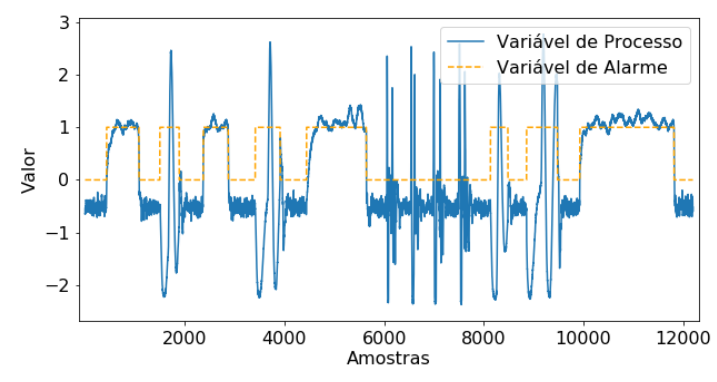

Figura 7. Variável XMEAS10 balanceada e escalada.

Após o balanceamento, a variável saiu de um valor de pouco mais de noventa mil amostras, para cerca de doze mil. Possuindo agora, metade dos seus pontos com alarme ativo e a outra metade desativo.

Após a aplicação do $K$-means nos dados de treinamento, os dados do banco de teste passam pela modelo para serem rotulados e assim gerar o sinal de alarme. Para o cálculo das métricas de avaliação é preciso a definição prévia dos alarmes esperados. Essa definição foi feita usando os mesmos critérios de geração de alarmes definidos na Seção 4.4 .

\section{RESULTADOS}

Aqui são mostrados os resultados obtidos na aplicação da abordagem proposta para a geração de sinais de alarmes utilizando os bancos de teste. Para os testes, algumas variáveis de processo foram selecionadas ao critério de mostrarem maior reação aos distúrbios. Tais foram, XMEAS01, XMEAS04 e XMEAS10. Para a avaliação do banco de teste, as métricas de FAR, MAR, AAD e chattering index descritos na Seção 2 foram utilizados.

A Figura 8 traz o resultado da aplicação do sistema de detecção de alarmes para a variável XMEAS10, com ocorrências aleatórias do Distúrbio 2.

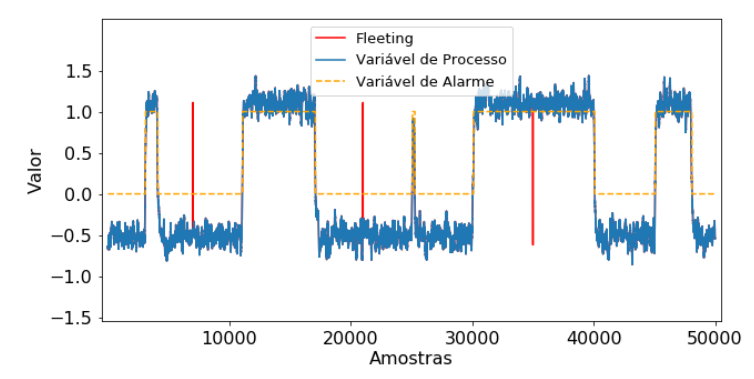

Figura 8. Resultado da detecção na Variável XMEAS10 com o Distúrbio 2.

Graficamente o resultado obtido se demonstra satisfatório. As regiões de fleeting alarms criadas artificialmente não geram alarmes incômodos ou mesmo desativaram um alarme legitimo. A Tabela 2 traz os resultados quantitativos gerais dos testes. Um único treinamento foi feito para cada variável, usando o banco de treinamento, já os testes de validação foram feitos para cada distúrbio separadamente. Podemos ressaltar ainda os baixos valores de $M A R, F A R$, $A A D$ e $C I$ para a variável XMEAS10.

Tabela 2. Resultados.

\begin{tabular}{cccccc} 
Var & Dist & MAR & FAR & AAD $(s)$ & Chattering \\
\hline 01 & 1 & 0,0023 & 0,0005 & 511,2 & $1 \times 10^{-9}$ \\
01 & 2 & 0,0 & 0,0 & - & 0,0 \\
01 & 6 & 0,5813 & 0,0005 & 6024 & $1 \times 10^{-9}$ \\
04 & 1 & 0,0 & 0,0109 & 918 & $5 \times 10^{-10}$ \\
04 & 2 & 0,0 & 0,0 & - & 0,0 \\
04 & 6 & 0,0 & 0,0 & - & 0,0 \\
10 & 1 & 0,0 & 0,0356 & - & $4 \times 10^{-9}$ \\
10 & 2 & 0,0006 & 0,0013 & 100,8 & $5 \times 10^{-10}$ \\
10 & 6 & 0,5911 & 0,0 & 6648 & $3 \times 10^{-10}$ \\
\hline
\end{tabular}

Os parâmetros de janela encontrados para as variáveis que resultaram em um melhor desemprenho pelas métricas, estão descritos na Tabela 3. Os valores das janelas foram encontrados baseados nos testes com os distúrbios que provocavam maiores reações para aquela variável. Notouse que os valores de $n$ eram sempre maiores que $m$. A variável 10 apresenta um valor de $n$ igual a 10 e $m$ igual a 9 que equivalem a aproximadamente a 6 minutos. Esse período, diferentemente de delay timers, não apresenta um atraso mínimo garantido. A variável 10 apresenta para o Distúrbio 2 um atraso de menos de 2 minutos.

Ao ocorrer a troca de distúrbio testado, é possível perceber um aumento considerável em algumas métricas de desempenho. Isso acontece devido à metodologia aplicada, onde 
Tabela 3. Parâmetros das janelas.

\begin{tabular}{ccc} 
Var & $\mathrm{n}$ & $\mathrm{m}$ \\
\hline 01 & 10 & 5 \\
04 & 9 & 5 \\
10 & 10 & 9
\end{tabular}

os tamanhos das janelas foram encontradas para prover melhores métricas na detecção do distúrbio que mais a afeta. É possível notar também que algumas variáveis não tinham reações alarmantes para aquele distúrbio, logo, não se tinha nenhum alarme a ser ativo, impossibilitando o cálculo de métricas.

Para a aplicação destes métodos, foi utilizada a biblioteca para análise de dados scikit-learn na linguagem de programação Python. Foram utilizados os parâmetros padrões do kmeans e um número de clusters igual a 2.

\section{CONCLUSÕES}

O trabalho tinha como proposta uma nova abordagem para detecção de alarmes em plantas industriais usando técnicas de aprendizado de máquina. É possível afirmar com base nos experimentos que a abordagem obteve êxito em detectar alarmes com bons valores para as métricas de desempenho. O baixo número de parâmetros a serem ajustados e a facilidade de encontrar valores adequados, são pontos positivos que foram alcançados. O desempenho dessa nova abordagem está relacionado as complexidades de treinamento e predição dos modelos escolhidos. Considerando o uso do K-means, o algoritmo de treinamento depende da convergência, enquanto o de predição depende do número de clusters $k$ e da dimensão da janela $w_{\text {ent }}$.

O tamanho escolhido para as janelas $w_{v a r}$ e $w_{a l r}$ e a disparidade entre seus valores, afetam o tempo de atraso na detecção. Como os comprimentos em amostras das janelas são muito pequenos em relação ao tamanho total do sinal, os valores de $w_{\text {ent }}$ levam um certo tempo até trocarem o valor da predição, já que em uma escala menor, a mudança de região não é abrupta. A forma como foi construída a variável de treinamento, com ocorrências de variados distúrbios ao longo do tempo, é um ponto a ser visto com cuidado, já que os distúrbios podem conter pontos com valores parecidos, dificultando para o sistema a distinção entre distúrbios.

Analisar mais afundo o comportamento dos pontos, de modo a conhecer melhor a forma como estão agrupados, para assim, fazer uso de outras técnicas de aprendizado de máquina que melhor se adéquem a natureza da variável de processo. Formular maneiras de encontrar parâmetros ótimos, como padrões entre os tamanhos das janelas e estudar qual melhores valores para $n$ e $m$ que se adéquam para detecção de cada distúrbio. Aplicar pré-processamento na variável de processo, fazendo uso de filtros que tornem esses valores mais fidedignos ao comportamento da variável. Estes são alguns pontos a serem estudados e mostrados em trabalhos futuros.

\section{REFERÊNCIAS}

Adnan, N.A., Izadi, I., and Chen, T. (2011). On expected detection delays for alarm systems with deadbands and delay-timers. Journal of Process Control, 21(9), 1318 1331.

ANSI/ISA (2009a). ANSI/ISA-18.2-2009, Management of Alarm Systems for the Process Industries. Technical report, American National Standard/Instrumentations, Systems \& Automation Society. (ANSI/ISA-2009).

ANSI/ISA (2009b). Management of Alarms Systems for the Process Industries. Standard, American National Standard/Instrumentations, Systems \& Automation Society. (ANSI/ISA-2009), North Carolina.

Bransby, M. and Jenkinson, J. (1998). Alarming performance. Computing Control Engineering Journal, 9(2), 61 -67. doi:10.1049/cce:19980203.

Downs, J.J. and Vogel, E.F. (1993). A plant-wide industrial process control problem. Computers \& chemical engineering, 17(3), 245-255.

Hugo, A.J. (2009). Estimation of alarm deadbands. IFAC Proceedings Volumes, 42(8), 663 - 667. 7th IFAC Symposium on Fault Detection, Supervision and Safety of Technical Processes.

Manca, D. and Brambilla, S. (2012). Dynamic simulation of the bp texas city refinery accident. Journal of Loss Prevention in the Process Industries, 25(6), 950 - 957.

Naghoosi, E., Izadi, I., and Chen, T. (2011). A study on the relation between alarm deadbands and optimal alarm limits. In Proceedings of the 2011 American Control Conference, 3627-3632. IEEE.

VanderPlas, J. (2016). Python Data Science Handbook: Essential Tools for Working with Data. O'Reilly Media, Inc., 1st edition.

Wang, J. and Chen, T. (2013). An online method for detection and reduction of chattering alarms due to oscillation. Computers \& Chemical Engineering, 54, $140-150$.

Wang, J. and Chen, T. (2014). An online method to remove chattering and repeating alarms based on alarm durations and intervals. Computers 8 Chemical Engineering, 67, 43-52.

Wang, J., Yang, F., Chen, T., and Shah, S.L. (2016). An overview of industrial alarm systems: main causes for alarm overloading, research status, and open problems. IEEE Transactions on Automation Science and Engineering, 13(2), 1045-1061.

Xu, J., Wang, J., Izadi, I., and Chen, T. (2012). Performance assessment and design for univariate alarm systems based on far, mar, and aad. IEEE Transactions on Automation Science and Engineering, 9(2), 296-307.

Zhang, W., Lin, X., and Su, X. (2010). Transport and fate modeling of nitrobenzene in groundwater after the songhua river pollution accident. Journal of Environmental Management, 91(11), 2378 - 2384. 